(C) 2022, The Authors. Published by Elsevier Inc. and Fass Inc. on behalf of the American Dairy Science Association ${ }^{\circledR}$. This is an open access article under the CC BY license (http://creativecommons.org/licenses/by/4.0/).

\title{
Effect of als $D$ deletion and overexpression of nox and alsS on diacetyl and acetoin production by Lacticaseibacillus casei during milk fermentation
}

\author{
Huaixiang Tian, ${ }^{1} \odot$ Yan Jing, ${ }^{1}$ Haiyan Yu, ${ }^{1} \odot$ Juan Huang, ${ }^{1}$ Haibin Yuan, ${ }^{1}$ Xinman Lou, ${ }^{1}$ Bei Wang, ${ }^{2} \odot$ \\ Zhiyuan $\mathrm{Xu}^{3}$ and Chen Chen ${ }^{1 *}$ (1) \\ ${ }^{1}$ School of Perfume and Aroma Technology, Shanghai Institute of Technology, Shanghai 201418, P.R. China \\ ${ }^{2}$ School of Food and Health, Beijing Technology and Business University, Beijing 100048, P.R. China \\ ${ }^{3}$ State Key Laboratory of Dairy Biotechnology, Shanghai Engineering Research Center of Dairy Biotechnology, Dairy Research Institute, \\ Bright Dairy and Food Co. Ltd., Shanghai 201418, P.R. China
}

\section{ABSTRACT}

Diacetyl and acetoin are key aroma components of fermented milk but are produced in low concentrations by starter cultures. In this study, we expressed NADH oxidase, acetolactate synthase, and inactivated acetolactate decarboxylase in Lacticaseibacillus casei TCS to generate recombinant $L$. casei strains, and investigated the effects of the genes encoding these enzymes on diacetyl and acetoin production during milk fermentation. In the single-gene recombinant strains tested, diacetyl concentrations were highest in milk fermented by L. casei TCSI-nox (nox gene overexpressed, 3.68 $\mathrm{mg} / \mathrm{kg}$ ), whereas acetoin concentrations were highest in milk fermented by L. casei TCS- $\Delta$ alsD (alsD gene deleted, $32.94 \mathrm{mg} / \mathrm{kg}$ ). Moreover, diacetyl and acetoin concentrations were higher in the inducible strains than in the corresponding constitutive strains (e.g., TCSInox vs. TCSC-nox, and TCSI- $\Delta a l s D-n o x$ vs. TCSC$\Delta$ alsD-nox). This phenomenon was also reflected in the protein expression levels and enzyme activities. In the double-gene recombinant strains tested, the highest concentrations of diacetyl and acetoin were produced by L. casei TCSI- $\Delta$ alsD-nox (nox overexpressed and alsD deleted, $4.66 \mathrm{mg} / \mathrm{kg}, 69.62 \mathrm{mg} / \mathrm{kg}$, respectively). The triple-gene recombinant $L$. casei TCS- $\Delta$ alsD-nox-als $S$ produced the highest concentrations of diacetyl and acetoin, which were 2.38 and 11.19 times, respectively, the concentrations produced by the original strain. These results show that the nox, als $S$, and alsD genes make key contributions to the biosynthesis of diacetyl and acetoin by $L$. casei. The modification of multiple genes had a synergistic effect, leading to greatly in-

Received August 16, 2021.

Accepted December 20, 2021.

*Corresponding author: chenchen@sit.edu.cn creased synthesis of diacetyl and acetoin by $L$. casei during its fermentation of milk.

Key words: fermented milk, gene knockout, overexpression, diacetyl, acetoin

\section{INTRODUCTION}

Fermented milk is a dairy product made by fermenting raw bovine, caprine, or ovine milk, or the corresponding powdered milk, with lactic acid bacteria (LAB) as the starter cultures (Tamime, 2002). Fermented milk is one of the most popular fermented dairy products globally, due to its texture, nutrition, and flavor (Shiby and Mishra, 2013; Chen et al., 2017). Flavor plays a vital role in determining consumers' acceptance of and preference for fermented milk (Güler, 2007). The acknowledged and key compounds contributing to the flavor profile of fermented milk are diacetyl and acetoin (Sodini et al., 2004), as these contribute the characteristic milky and buttery flavors to fermented milk (Cheng, 2010; Chiocchetti et al., 2019).

Diacetyl and acetoin can be synthesized by microorganisms during fermentation (Sieuwerts et al., 2008; Chen et al., 2017; Figure 1). The biosynthetic precursors for diacetyl and acetoin are usually citric acid and glucose. Glucose is converted to pyruvate via glycolysis, whereas citric acid produces oxaloacetic acid by the catalytic action of citrate glucose and then pyruvate is generated by decarboxylation (Smit et al., 2005). Acetolactate synthase (ALS) then catalyzes the synthesis of $\alpha$-acetolactate from pyruvate (Zhang et al., 2013). Diacetyl is generated from $\alpha$-acetolactate through nonenzymatic oxidative decarboxylation under acidic conditions. Acetoin is generated either from $\alpha$-acetolactate by $\alpha$-acetolactate decarboxylase or from diacetyl by diacetyl reductase. Diacetyl and acetoin are either excreted as end products or partially reduced to butanediol catalyzed by acetoin reductase (Papagianni, 2012; Yang et al., 2017). The application and development of genomic and metabolomic technologies have enabled 


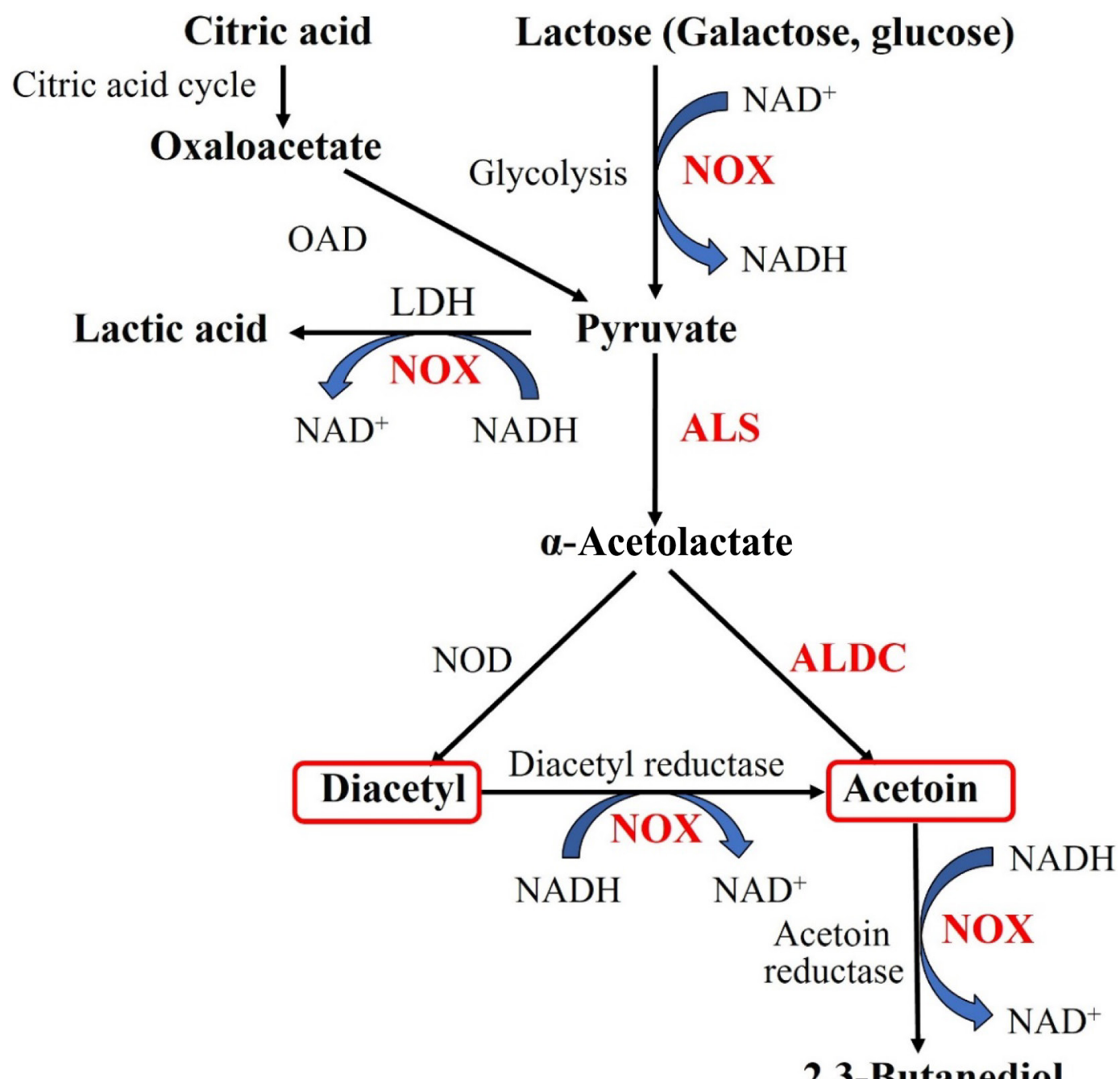

2,3-Butanediol

Figure 1. Simplified pathway of diacetyl and acetoin biosynthesis in lactic acid bacteria. NOX $=$ NADH oxidase; ALS $=$ acetolactate synthase; ALDC = acetolactate decarboxylase LDH = lactate dehydrogenase; OAD = oxaloacetate decarboxylase; NOD = non-enzymatic oxidative decarboxylation. Red boxes represent the key flavor compounds; words in red represent key enzymes in the biosynthetic pathway.

study of the key genes involved in the biosynthesis of diacetyl and acetoin (Liu et al., 2008; Sieuwerts et al., 2010).

There is current interest in enhancing the flavor of food by the endogenous generation of flavor compounds rather than by the exogenous addition of such compounds. Because the flavor of fermented milk is mainly produced by metabolism of LAB, their production of diacetyl and acetoin has attracted much research attention (Lo et al., 2018). The genera Lactococcus, Leuconostoc, Streptococcus, Lactobacillus, and Bifidobacterium (Liu et al., 2011; D'Angelo et al., 2017; Wang et al., 2019b; Raveschot et al., 2020) have all shown potential to produce diacetyl and acetoin during fermented milk production. In a previous study, we used a high-throughput screening method to identify Lacticaseibacillus casei TCS, an L. casei strain that has high diacetyl and acetoin production capacity (Tian et al., 2019). Thus L. casei TCS can be used as a parent strain for genetic modification and as a starter culture to improve the aromatic profile of fermented milk.

To increase the production of diacetyl and acetoin by L. casei during fermented milk production, this study examined the influences of 3 genes: nox, als $S$, and alsD. Specifically, overexpression and gene knockout strategies were used to make single- and multiple-gene modifications to determine the influences and synergistic effects of these genes on diacetyl and acetoin production. The results of this study clarify the biosynthesis of diacetyl and acetoin by $L$. casei and provide a theoretical 
basis for the future optimization of flavor production in fermented milk.

\section{MATERIALS AND METHODS}

\section{Bacterial Strains, Plasmids, and Culture Conditions}

The strains and plasmids used in this study are listed in Table 1. Lacticaseibacillus casei TCS was used as the host strain for genetic modification, and Escherichia coli TOP10 was used as the host for cloning. Lacticaseibacillus casei TCS and the recombinant strains were cultured in de Man, Rogosa, and Sharpe (MRS) broth (Merck) at $37^{\circ} \mathrm{C}$ without aeration. The E. coli strains were cultured in Luria Bertani broth (Merck) at $37^{\circ} \mathrm{C}$ with aeration at $200 \mathrm{rpm}$. When appropriate, the following antibiotics were added: $200 \mu \mathrm{g} / \mathrm{mL}$ erythromycin and $50 \mu \mathrm{g} / \mathrm{mL}$ kanamycin for E. coli, and 10 $\mu \mathrm{g} / \mathrm{mL}$ erythromycin for recombinant $L$. casei strains, as described in our previous studies (Chen et al., 2017, 2020). The primers used in this study are listed in Table 2.

\section{Construction of Recombinant Strains Overexpressing the nox and alsS Genes}

The nox gene was amplified by PCR using Streptococcus mutans chromosomal DNA as a template, due to the high NADH oxidase activity of this strain (Li et al., 2018). The alsS gene was amplified by PCR using L. casei TCS chromosomal DNA as a template. The constructed recombinant strains had either constitutive or inducible expression. In those with constitutive expression, the nox and als $S$ genes were amplified using the primer pairs nox- $\mathrm{F}_{\mathrm{p}} /$ nox $_{\mathrm{p}}$ and alsS- $\mathrm{F}_{\mathrm{p}} /$ alsS $_{\mathrm{p}} \mathrm{R}_{\mathrm{p}}$, respectively. The PCR products were purified using the TIANquick Midi Purification Kit (Tiangen Biotech Co. Ltd.) and then cloned into plasmid pIB184 using a One Step Cloning Kit (Vazyme Biotech Co. Ltd.) to construct a constitutive expression plasmid. After transformation into $E$. coli, the constructs were verified by PCR using the primers pIB184-F and pIB184-R, and their construction was confirmed by DNA sequencing. The constructed plasmids were named pIB184-nox and pIB184-alsS. The recombinant plasmids were then

Table 1. Strains and plasmids used in this study

\begin{tabular}{|c|c|c|}
\hline Strains and plasmids & Relevant characteristics $^{1}$ & Source $^{2}$ \\
\hline \multicolumn{3}{|l|}{ Strains } \\
\hline \multicolumn{3}{|l|}{ Escherichia coli } \\
\hline E. coli DH5 $\alpha$ & Cloning host & Promega \\
\hline E. coli Top10 & Cloning host & Takara \\
\hline Top/p1628-RFP & Kana-r, E. coli Top10 containing p1628-RFP & Song et al., 2017 \\
\hline DH5 $\alpha /$ nox -409 & Em-r, E. coli DH5 $\alpha$ containing pSIP409-nox & This work \\
\hline $\mathrm{DH} 5 \alpha /$ nox -184 & Em-r, E. coli DH5 $\alpha$ containing pIB184-nox & This work \\
\hline $\mathrm{DH} 5 \alpha /$ alsS-409 & Em-r, E. coli DH5 $\alpha$ containing pSIP409-alsS & This work \\
\hline $\mathrm{DH} 5 \alpha / a l s S-184$ & Em-r, E. coli DH5 $\alpha$ containing pIB184-alsS & This work \\
\hline Top10/Up-Down-gRNA & Kana-r, E. coli Top10 containing p-Up-Down-gRNA & This work \\
\hline \multicolumn{3}{|l|}{ Lacticaseibacillus casei } \\
\hline TCS & Wild-type Lacticaseibacillus casei & CCTCC no. M2017364 \\
\hline TCS- $\Delta$ alsD & In-frame deletion of alsD in L. casei TCS & This work \\
\hline TCSI-nox & Em-r, L. casei TCS containing pSIP409-nox plasmid & This work \\
\hline TCSC-nox & Em-r, L. casei TCS containing pIB184-nox plasmid & This work \\
\hline TCSI- alsS & Em-r, L. casei TCS containing pSIP409-alsS plasmid & This work \\
\hline TCSC-alsS & Em-r, L. casei TCS containing pIB184-alsS plasmid & This work \\
\hline TCSI- $\Delta$ alsD-nox & Em-r, L. casei TCS- $\Delta$ alsD containing pSIP409-nox plasmid & This work \\
\hline TCSC- $\Delta$ alsD-nox & Em-r, L. casei TCS- $\Delta$ alsD containing pIB184-nox plasmid & This work \\
\hline TCSI- $\Delta$ alsD-alsS & Em-r, L. casei TCS- $\Delta$ alsD containing pSIP409-alsS plasmid & This work \\
\hline TCSC- $\Delta a l s D-a l s S$ & Em-r, L. casei TCS- $\Delta$ alsD containing pIB184-alsS plasmid & This work \\
\hline TCS- $\Delta$ alsD-nox-als $S$ & Em-r, L. casei TCS- $\Delta$ alsD containing pSIP409-nox-alsS plasmid & This work \\
\hline \multicolumn{3}{|c|}{ 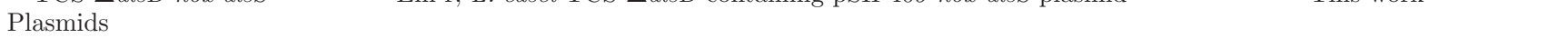 } \\
\hline pSIP409 & Em-r, cloning vector derived from the plasmid pSIP401 & Sørvig et al., 2005 \\
\hline pIB184 & Em-r, cloning vector & Li et al., 2015 \\
\hline pLCNICK-1628 & Kana-r, cloning vector derived from the plasmid pIB184 & Song et al., 2017 \\
\hline pSIP409-nox & Em-r, pSIP409 derivative, gusA replaced by nox & This work \\
\hline pIB184-nox & Em-r, pIB184 derivative, gusA replaced by nox & This work \\
\hline pSIP409-alsS & Em-r, pSIP409 derivative, gus $A$ replaced by als $S$ & This work \\
\hline pIB184-als $S$ & Em-r, pIB184 derivative, gusA replaced by als $S$ & This work \\
\hline pSIP409-nox-alsS & Em-r, pSIP409 derivative, gusA replaced by nox and als $S$ & This work \\
\hline p-Up-Down-gRNA & $\begin{array}{l}\text { Plasmid derived from PCR inserts containing upstream and downstream } \\
\text { alsD genes and targeting RNA }\end{array}$ & This work \\
\hline
\end{tabular}

${ }^{1}$ Kana-r = kanamycin resistant; Em-r = erythromycin resistant.

${ }^{2}$ Promega, Madison, WI; Takara, Tokyo, Japan; CCTCC = China Center for Type Culture Collection, Wuhan, China. 
transformed into competent $L$. casei TCS cells by electroporation. The recombinant strains were verified by PCR with the corresponding primers (pIB184-F and pIB184-R) and named L. casei TCSC-nox and L. casei TCSC-alsS.

Similarly, to generate recombinant bacteria with inducible expression, the nox and alsS genes were amplified using the primer pairs nox- $\mathrm{F}_{4} /$ nox- $\mathrm{R}_{4}$ and alsS- $\mathrm{F}_{4} /$ alsS- $\mathrm{R}_{4}$, respectively. Inducible expression plasmids were then constructed by cloning the PCR products into plasmid pSIP409 using the One Step Cloning Kit (Vazyme). Transformation was performed according to the method described previously. The resulting plasmids, named pSIP409-nox and pSIP409-alsS, were verified by PCR using the primers $403-\mathrm{F}$ and $403-\mathrm{R}$. The recombinant plasmids were then transformed into $L$. casei TCS cells, and their transformation was verified by repeating the steps previously described. The resulting recombinant strains were named $L$. casei TCSI-nox and L. casei TCSI-alsS.

\section{Construction of Recombinant Strain with the alsD Gene Knocked Out}

A knockout vector pLCNICK-1628 was constructed using the clustered regularly interspaced short palin- dromic repeats (CRISPR) gene editing system, according to the method described by Song et al. (2017), with some modifications.

The pLCNICK-1628 plasmid was double-digested with endonucleases ApaI and XbaI, and the resulting vector fragment was recovered by electrophoretic separation and gel excision. The upstream and downstream sequences of the $L$. casei TCS alsD gene were amplified by PCR using $L$. casei TCS DNA as a template. The sgRNA fragment was obtained by PCR amplification of pLCNICK-1628, using the primers 2013gRNA-F and 2013gRNA-R. The upstream and downstream sequences and the sgRNA fragment were ligated to the digested vector fragment using the One Step Cloning Kit (Vazyme). After transformation into E. coli TOP10, plasmids were extracted from positive transformants through colony PCR, and verified by enzymatic digestion and sequencing. The transformed plasmid was named p-Up-Down-gRNA, and the recombinant strain was named E. coli TOP10/Up-Down-gRNA. The recombinant plasmid, p-Up-Down-gRNA, was then electrotransformed into competent $L$. casei TCS cells. Colony PCR with the primer pairs 2013yz-F/2013yz-R and TCSyz-F/TCSyz-R was used to verify that the recombinant plasmid had been incorporated into $L$. casei TCS and that the alsD gene had been knocked out of

Table 2. Primers used in this study; $\mathrm{F}=$ forward; $\mathrm{R}=$ reverse

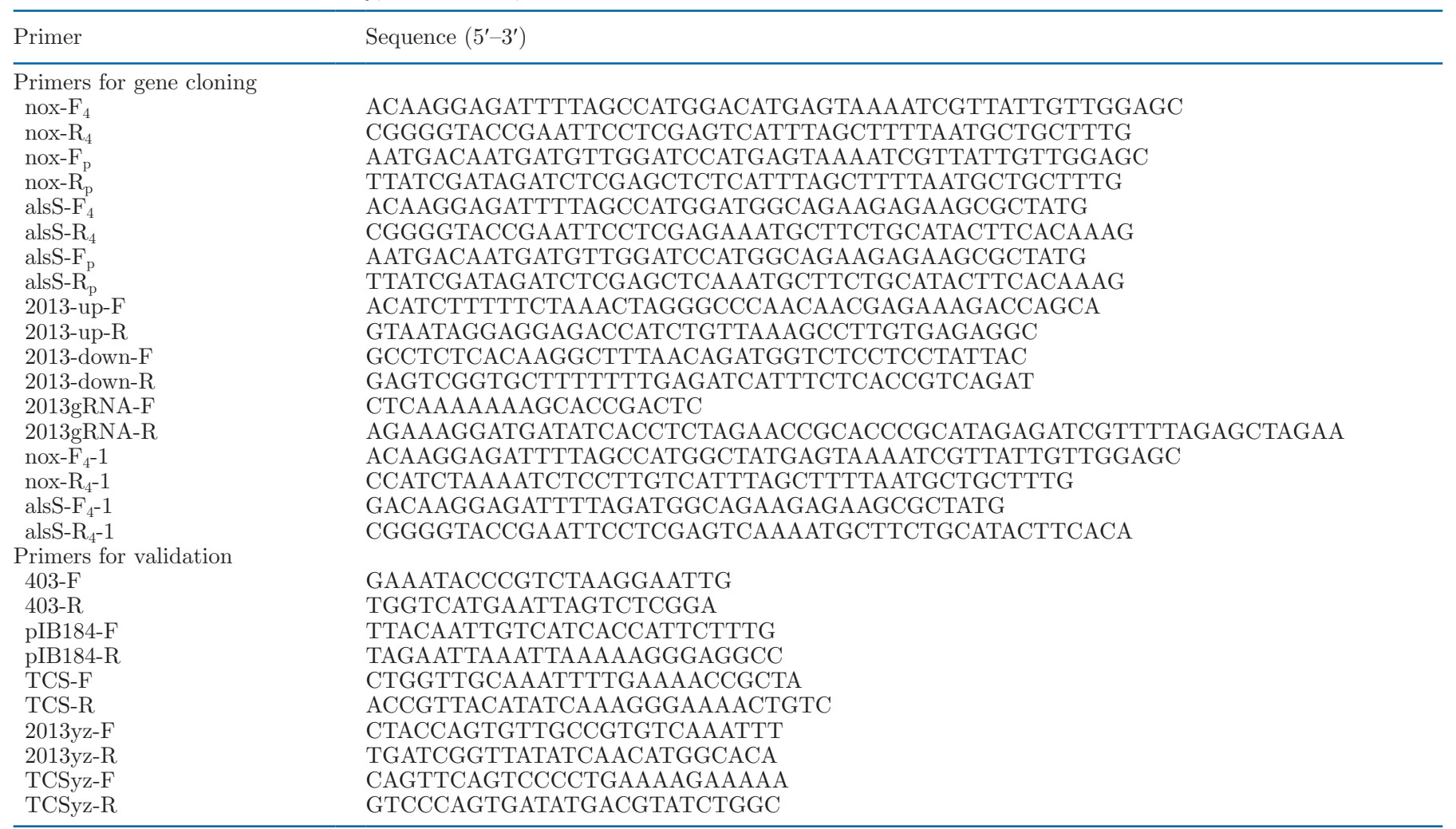


the genome of $L$. casei TCS. The knockout strain was named L. casei TCS- $\Delta$ alsD.

\section{Construction of Recombinant Strains of L. casei with Multiple-Gene Modifications}

To build recombinant strains with a single-gene overexpression based on alsD gene deletion, the recombinant plasmids (pSIP409-nox, pSIP409-alsS, pIB184-nox, and pIB184-alsS) described earlier were transformed by electroporation into competent cells of TCS- $\Delta$ alsD, an L. casei TCS-derived alsD-knockout strain. Recombinant strains were selected and named TCSI- $\Delta$ alsD-nox, TCSC- $\Delta$ alsD-nox, TCSI- $\Delta$ alsD-als $S$, and TCSC- $\Delta$ alsD-alsS.

To coexpress the nox and als $S$ genes in a single vector based on als $D$ gene deletion, a ribosome-binding site was introduced into the $5^{\prime}$-terminus of the als $S$ gene, and the nox and als $S$ fragments (generated using the primer pairs nox- $\mathrm{F}_{4}-1 /$ nox $_{-} \mathrm{R}_{4}-1$ and alsS- $F_{4}-1 /$ alsS- $\mathrm{R}_{4}-1$, respectively) were then ligated into a vector (pSIP409) using the One Step Cloning Kit (Vazyme). The recombinant plasmid was electrotransformed into competent TCS- $\Delta$ alsD cells, as described previously, and the recombinant strain was verified by sequencing. The constructed strain that coexpressed the nox and alsS genes and had its alsD gene knocked out was named $L$. casei TCS- $\Delta$ alsD-nox-alsS.

\section{Expression of Recombinant Bacterial Proteins}

Protein expression was performed according to our previously described method (Chen et al., 2014). Recombinant strains with inducible gene expression (TCSI-nox, TCSI- $\Delta$ alsD-nox, TCSI- als $S$, TCSI- $\Delta$ alsD-als $S$, and TCS- $\Delta$ alsD-nox-alsS) were inoculated into 100 $\mathrm{mL}$ of MRS broth containing $10 \mu \mathrm{g} / \mathrm{mL}$ erythromycin, and the resulting mixture was cultured at $37^{\circ} \mathrm{C}$ until it reached an optical density value at $600 \mathrm{~nm}\left(\mathbf{O D}_{600}\right)$ of 0.3 to 0.5 . An inducer (IP-673) was added to the culture to give a concentration of $25 \mathrm{ng} / \mathrm{mL}$, and the culture was incubated at $37^{\circ} \mathrm{C}$ until an $\mathrm{OD}_{600}$ value of 1.5 was reached. After induction, the cells were harvested by centrifugation $\left(8,000 \times \mathrm{g}, 4^{\circ} \mathrm{C}, 5 \mathrm{~min}\right)$, washed with sterile buffer (50 $\mathrm{m} M$ Tris $/ \mathrm{HCl}, \mathrm{pH} 8.0)$, resuspended in the same buffer supplemented with $25 \mathrm{mg} / \mathrm{mL}$ lysozyme, and then sonicated. Cellular debris was removed by centrifugation $\left(12,000 \times g, 4^{\circ} \mathrm{C}, 10 \mathrm{~min}\right)$, and the supernatant containing the soluble proteins was collected. The whole culture, including the supernatant and cell pellets before and after induction, was collected, and the expression of the recombinant proteins NADH oxidase (NOX) and ALS, was analyzed via SDS-PAGE $(12.0 \%, \mathrm{wt} / \mathrm{vol})$. Various IP-673 concentrations (25 and
$50 \mathrm{ng} / \mathrm{mL})$, induction temperatures $\left(16,25\right.$ and $\left.37^{\circ} \mathrm{C}\right)$, and induction times $\left(\mathrm{OD}_{600}=1.2,1.5\right.$, and 1.8) were used to determine which conditions led to the optimal expression of NOX and ALS.

\section{Enzyme Activity Determination}

Activity of ALS was determined according to the method described by Holtzclaw and Chapman (Holtzclaw and Chapman, 1975), with some modifications. Briefly, the assay was performed in a mixture comprising $1 \mathrm{~mL}$ of $1 \mathrm{mM}$ thiamine pyrophosphate, 5 $\mathrm{m} M \mathrm{MgCl}_{2}, 0.1 \mathrm{~m} M$ flavin adenine dinucleotide, $100 \mathrm{~m} M$ sodium pyruvate, and $300 \mu \mathrm{L}$ of crude enzyme solution. This mixture was heated at $37^{\circ} \mathrm{C}$ for $1 \mathrm{~h}$, at which time $200 \mu \mathrm{L}$ of $3 \mathrm{M} \mathrm{H}_{2} \mathrm{SO}_{4}$ was immediately added to terminate the reaction. After decarboxylation in a water bath at $60^{\circ} \mathrm{C}$ for $15 \mathrm{~min}, 8.5 \mathrm{~mL}$ of a coloring solution (5\% $\alpha$-naphthol, $0.5 \%$ creatine, $5 \%$ sodium hydroxide, and deionized water) was added. The resulting mixture was incubated for an additional $30 \mathrm{~min}$ at $37^{\circ} \mathrm{C}$ to allow for the acid hydrolysis of acetolactate to acetoin. Finally, the absorbance was measured at $520 \mathrm{~nm}$ using a spectrophotometer (Unico Instrument Co. Ltd.). One unit of enzyme activity is defined as the amount of enzyme that converts $1 \mathrm{nmol}$ of substrate into product in 1 min under these conditions (Holtzclaw and Chapman, 1975).

Activity of NOX was measured using an NADH Oxidase Activity Detection Kit (Solarbio Science and Technology). One unit of enzyme activity is defined as a change in the $600-\mathrm{nm}$ absorbance of a reaction system of 0.01 per min per 10,000 bacterial cells (Kawai et al., 2004).

\section{Preparation of Fermented Milk}

Fermented milk was produced using a previously described method, with some modifications (Tian et al., 2020). Various L. casei strains were revitalized in MRS broth and routinely cultured for 12 to $16 \mathrm{~h}$ at $37^{\circ} \mathrm{C}$. The cells were then harvested by centrifugation at $6,000 \times$ $g$ for 10 min at room temperature, washed twice with sterile water, and then resuspended in a volume of sterile water equal to the volume of the medium. The resulting suspension was used as the starter culture.

A skim milk solution was prepared with $12 \%$ (wt/ vol) skim milk powder (Fonterra), and the reconstituted milk was pasteurized according to the method described by Fernandez-Garcia and McGregor (1994). The milk was inoculated with $4 \%$ (wt/vol) starter cultures of L. casei strains and stirred thoroughly. Cells of the inducible recombinant strains (TCSI- nox, TCSIalss, TCSI- $\Delta a l s D-n o x$, TCSI- $\Delta a l s D$-als $S$, and TCS- 
$\Delta$ alsD-nox-alsS) were induced by adding IP-673 to give a final concentration of $25 \mathrm{ng} / \mathrm{mL}$. After inoculation, the samples were sealed in 100-mL glass bottles using polypropylene caps. The bottles were transferred to a thermostatically controlled water bath $\left(37^{\circ} \mathrm{C}\right)$, where they were incubated until they reached a $\mathrm{pH}$ of 4.6 . The resulting fermented samples were transferred to a refrigerator set to $4^{\circ} \mathrm{C}$ for ripening.

\section{Determination of Diacetyl and Acetoin Concentrations}

Gas chromatography spectrometry was used to measure diacetyl and acetoin concentrations, as described previously (Tian et al., 2017), with minor modifications. After ripening for $24 \mathrm{~h}, 5 \mathrm{~g}$ of a fermented milk sample was placed in a $15-\mathrm{mL}$ extraction bottle equipped with a rotor, and $10 \mu \mathrm{L}$ of 2 -octanol (internal standard, 19 $\mathrm{mg} / \mathrm{L}$ ) was added. The resulting mixture was balanced in a magnetic constant-temperature water bath at $55^{\circ} \mathrm{C}$ for $5 \mathrm{~min}$, and the extraction head was then inserted for $40 \mathrm{~min}$ to enrich the volatile substances in the headspace. The chromatographic and mass spectrometric conditions for subsequent sample detection were the same as those previously described. All analyses were performed in triplicate.

\section{Statistical Analysis}

The presented data are representative of 3 independent experiments. Statistical analyses were performed by one-way ANOVA (with Duncan's multiple range method) using SPSS (version 19.0; IBM Corp.). Differences with $P<0.05$ were considered statistically significant.

\section{RESULTS}

\section{Construction and Verification of Recombinant L. casei Strains}

The inducible plasmid pSIP409 and the constitutive plasmid pIB184 were used to construct inducible and constitutive expression vectors, respectively, for the nox and als $S$ genes. Thus, 4 recombinant strains of $L$. casei with a single expressed gene were constructed; these were named TCSI-nox, TCSC-nox, TCSI-alsS, and TCSC-alsS. The alsD gene was knocked out using the clustered regularly interspaced short palindromic repeats (CRISPR) gene editing system, and the resulting recombinant $L$. casei strain was named TCS- $\Delta$ alsD (Table 1).

Recombinant strains with multiple genetic modifications were then constructed based on L. casei TCS-
$\Delta a l s D$. Four strains of recombinant L. casei were constructed with double genetic modifications; these were named TCSI- $\Delta$ alsD-nox, TCSC- $\Delta$ alsD-nox, TCSI- $\Delta a l s D-a l s S$, and TCSC- $\Delta$ alsD-als $S$ (Table 1). A recombinant strain that coexpressed nox and als $S$ and had its alsD gene knocked out was also constructed; this was named $L$. casei TCS- $\Delta$ alsD-nox-alsS. In total, 10 mutant strains were constructed, and the enzyme activity and diacetyl and acetoin yields of these mutant strains were measured and compared with those of the wild-type strain.

\section{Protein Expression Levels of Recombinant L. casei Strains}

The recombinant strains with inducible expression vectors, TCSI-nox, TCSI- $\Delta$ alsD-nox, TCSI-alsS, TCSI$\Delta a l s D-a l s S$, and TCS- $\Delta$ alsD-nox-als $S$, were cultured in MRS broth until their $\mathrm{OD}_{600}$ values reached 0.6. Induced and non-induced cells were harvested in the late stationary phase, and the cell extracts were analyzed by SDS-PAGE. The optimal induction conditions for the 4 recombinant strains were a temperature of $16^{\circ} \mathrm{C}$ and a final IP-673 concentration of $50 \mathrm{ng} / \mathrm{mL}$. However, the optimal induction time varied between different strains. When L. casei TCSI-nox was induced for $59 \mathrm{~h}$ and $L$. casei TCSI- $\Delta$ alsD-nox was induced for $42 \mathrm{~h}$, an intense protein band was seen at 45 to $60 \mathrm{kDa}$ (Figure 2a, b), which is close to the expected size of recombinant NOX. The optimal induction times for L. casei TCSIalsS and L. casei TCSI- $\Delta$ alsD-als $S$ were $60 \mathrm{~h}$ and $44 \mathrm{~h}$, respectively (Figure 2c, d). The 2 recombinant strains expressing ALS also showed bands close to their expected size $(58.87 \mathrm{kDa})$. The optimal induction conditions for the recombinant L. casei coexpressing NOX and ALS (TCS- $\Delta$ alsD-nox-alsS) were $37^{\circ} \mathrm{C}$ and a final IP-673 concentration of $25 \mathrm{ng} / \mathrm{mL}$. This recombinant strain also showed unique bands at the expected sizes of $47.80 \mathrm{kDa}(\mathrm{NOX})$ and $63.76 \mathrm{kDa}$ (ALS; Figure 2e).

\section{Enzyme Activity of Wild-Type and Recombinant Strains}

The results of NOX and ALS activity measurements are shown in Figure 3. The NOX activities of the recombinant strains, TCSI-nox, TCSC-nox, TCSI- $\Delta$ alsDnox, TCSC- $\Delta$ alsD-nox, and TCS- $\Delta$ alsD-nox-als $S$, were $139.34,13.66,95.08,9.78$, and 8.63 times those of the wild-type strain, respectively. Activity of NOX was significantly higher in the recombinant strains with inducible NOX overexpression (TCSI- nox and TCSI$\Delta a l s D-n o x)$ than in those with constitutive NOX expression (TCSC-nox and TCSC- $\Delta$ alsD-nox; $P<0.05$ ). The ALS activity was also higher in the recombinant 

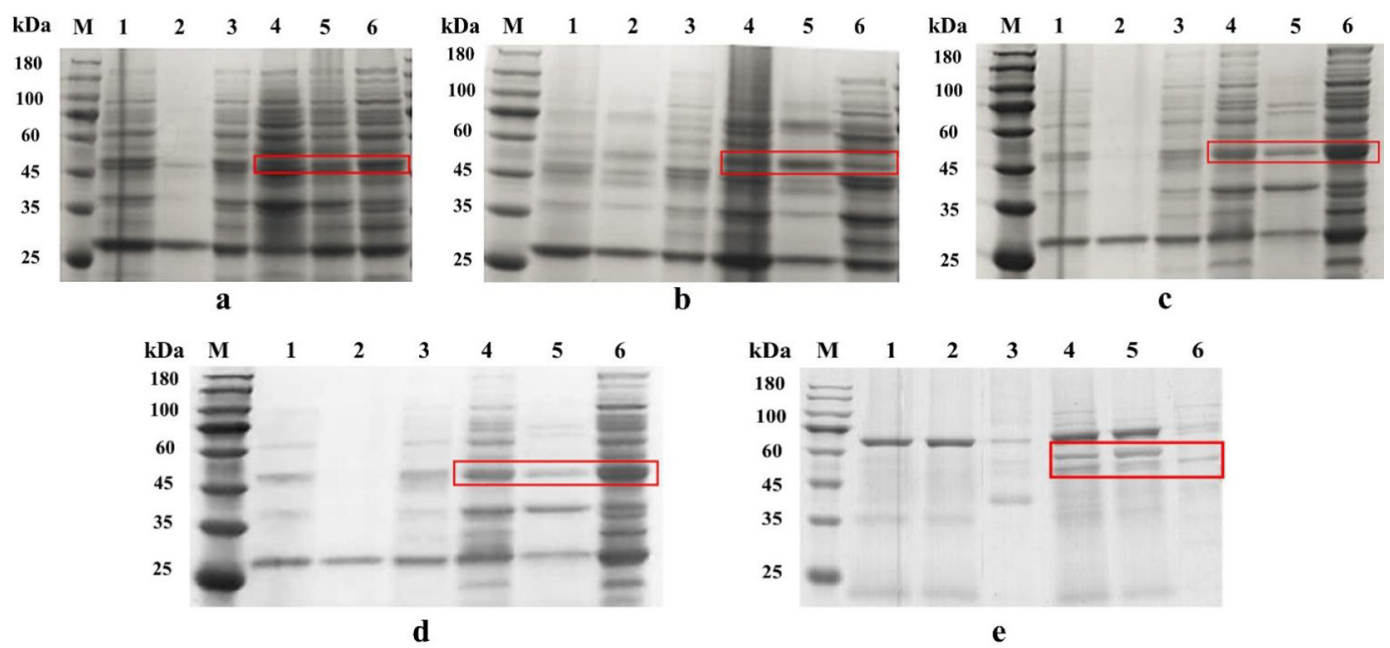

Figure 2. Expression of recombinant proteins in various Lacticaseibacillus casei TCS. (1) NADH oxidase (NOX) expression in TCSI-nox (a) and TCSI- $\Delta$ alsD-nox (b), and acetolactate synthase (ALS) expression in TCSI-alsS (c) and TCSI- $\Delta$ alsD-als $S$ (d), as analyzed by SDS-PAGE. The optimal induction conditions for the 4 recombinant strains were $16^{\circ} \mathrm{C}$ and a final IP- 673 concentration of $50 \mathrm{ng} / \mathrm{mL}$. (2) NOX and ALS expression in TCS- $\Delta$ alsD-nox-alsS (e), as analyzed by SDS-PAGE. The optimal induction conditions for the recombinant strain coexpressing NOX and ALS (TCS- $\Delta$ alsD-nox-als $S$ ) were $37^{\circ} \mathrm{C}$ and a final IP- 673 concentration of $25 \mathrm{ng} / \mathrm{mL} . \mathrm{M}=$ protein marker; 1,2 , and $3=$ whole culture, supernatant, and cell pellets before induction, respectively; 4,5 , and $6=$ whole culture, supernatant, and cell pellets after induction with IP-673, respectively; red box indicates the expected band. Strains are described in Table 1.

strains than in the original strain. However, although some differences were observed between the mutant and wild-type strains, enzyme activity did not significantly differ between the 5 recombinant strains (TCSC-alsS, TCSI- als $S$, TCSC- $\Delta a l s D-a l s S$, TCSI- $\Delta a l s D-a l s S$, and TCS- $\Delta$ alsD-nox-alsS; $P>0.05)$.

\section{Comparison of Diacetyl and Acetoin Yield in Milk Fermented by Various Recombinant L. casei Strains}

Ten recombinant L. casei strains (TCS- $\Delta a l s D$, TCSI-nox, TCSC-nox, TCSI-alsS, TCSC-alsS, TCSI$\Delta$ alsD-nox, TCSC- $\Delta$ alsD-nox, TCSI- $\Delta$ alsD-als $S$, TCSC- $\Delta$ alsD-alsS, and TCS- $\Delta$ alsD-nox-als $S$ ) and the wild-type strain (TCS) were used as starters for fermented milk production. Aromatic compounds were extracted from these samples, and diacetyl and acetoin were quantified by GC (Figure 4).

In general, the milk samples fermented by the mutant strains had higher concentrations of diacetyl and acetoin than those fermented by the wild-type strain. Of the strains with a single-gene modification, L. casei TCSI-nox, which had inducible nox gene overexpression, produced the highest diacetyl concentration (1.20 times the diacetyl concentration compared with strain TCS, Figure 4a). The acetoin concentration (Figure $4 \mathrm{~b}$ ) was highest in milk fermented by the recombinant L. casei TCS- $\triangle$ alsD, which had alsD knocked out. Milk fermented by this strain had 3.99 times the acetoin concentration of milk fermented by strain TCS. Milk fer- mented by either of the 2 strains with inducible expression (TCSI-nox and TCSI- alsS) had higher diacetyl and acetoin concentrations than milk fermented by either of the 2 strains with constitutive expression (TCSC-nox and TCSC-alsS). For example, the diacetyl and acetoin concentrations in milk fermented with the recombinant L. casei TCSI-nox (with inducible nox gene expression) were 1.15 times and 1.28 times, respectively, those in milk fermented by L. casei TCSC-nox (with constitutive nox gene expression).

In strains with a double-gene modification, the diacetyl and acetoin concentrations were highest in milk fermented by $L$. casei TCSI- $\Delta$ alsD-nox, which had inducible nox gene expression and the alsD gene knocked out. The diacetyl and acetoin concentrations in milk fermented by this recombinant strain were 1.52 and 8.43 times, respectively, those in milk fermented by strain TCS. Furthermore, the acetoin concentrations were significantly higher in milk fermented by doublegene recombinant strains than in milk fermented by single-gene recombinant strains. For example, the acetoin concentration in milk fermented by $L$. casei TCSI- $\Delta$ alsD-nox, which had inducible nox gene expression and the alsD gene knocked out, was 1.31 times the sum of the acetoin concentration in milk fermented by $L$. casei TCS- $\Delta$ alsD (with alsD gene deletion) and $L$. casei TCSI-nox (with inducible nox gene expression). Lacticaseibacillus casei TCS- $\Delta$ alsD-nox-als $S$, which had 3 genetic modifications, yielded the highest concentrations of diacetyl and acetoin of all tested strains, with 


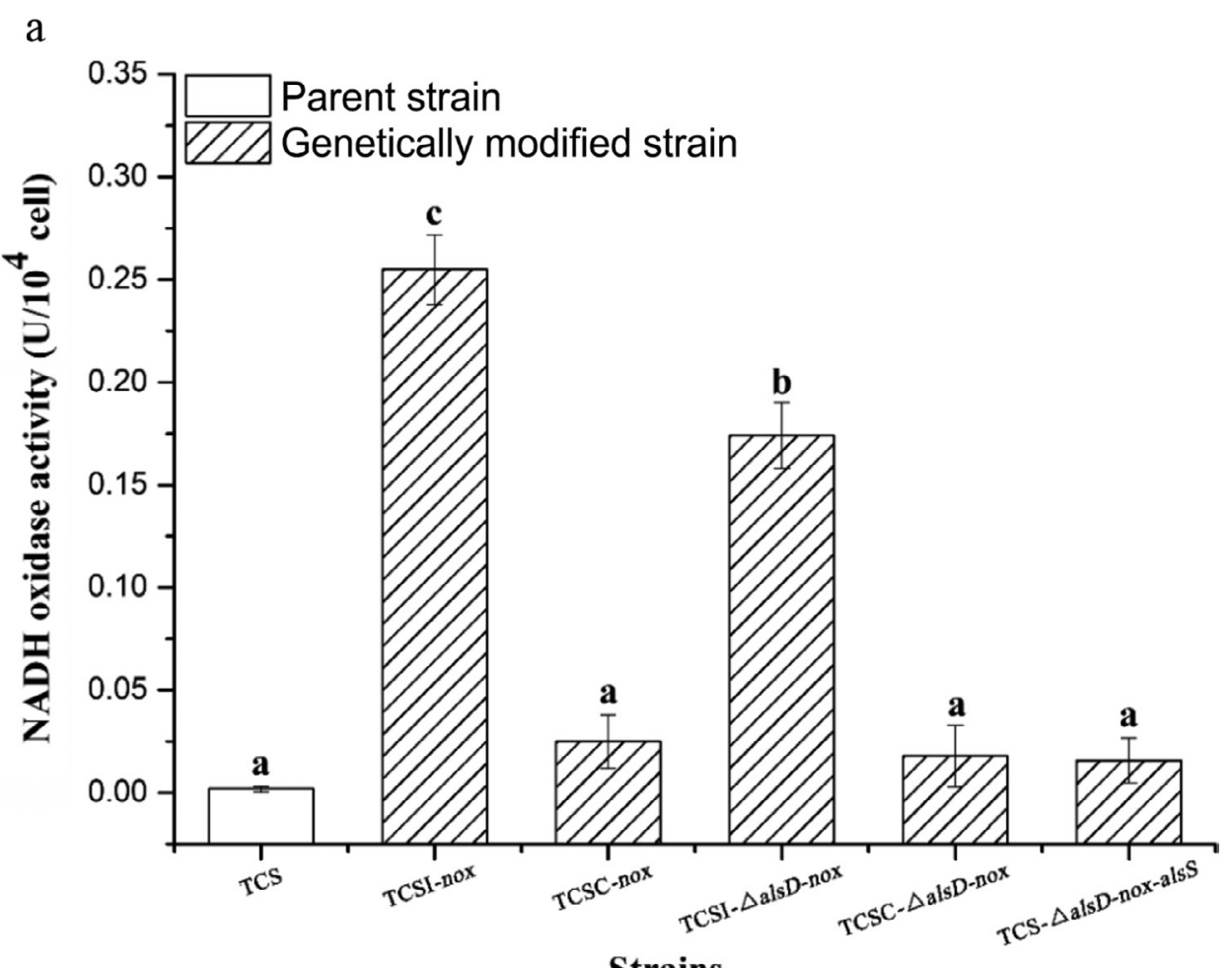

Strains

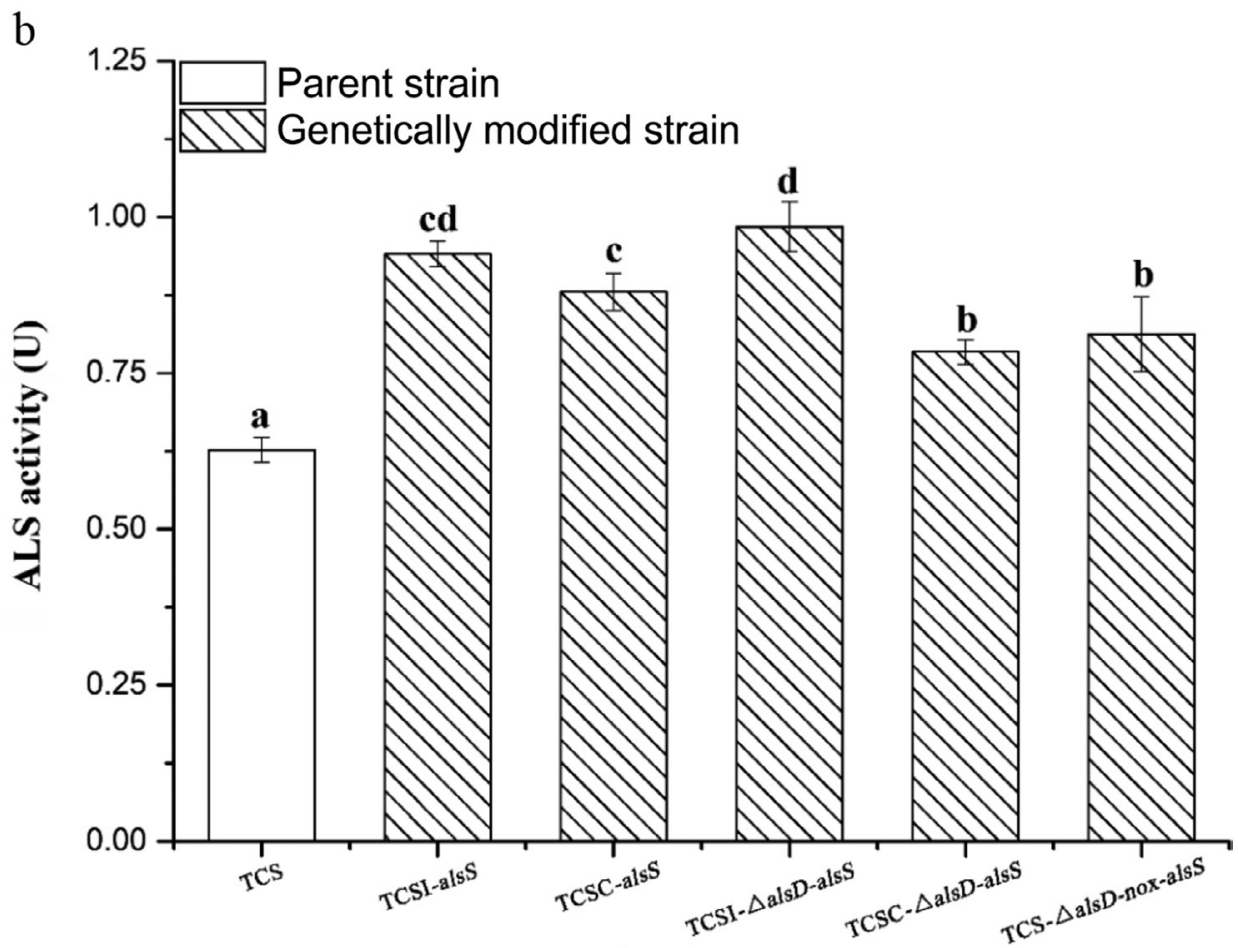

Strains

Figure 3. Activities of NADH oxidase (a) and acetolactate synthase (b) of wild-type and mutant strains of Lacticaseibacillus casei TCS. Strains are described in Table 1. One unit (U) of acetolactate synthase enzyme activity is defined as the amount of enzyme that converts 1 nmol of substrate into product in $1 \mathrm{~min}$. One unit (U) of NADH oxidase enzyme activity is defined as a change in the absorbance at $600 \mathrm{~nm}\left(\mathrm{~A}_{600}\right)$ of a reaction system of 0.01 per min per 10,000 bacterial cells. Error bars represent SD based on 3 replicates. Values marked with different letters (a-d) are significantly different $(P<0.05)$. 


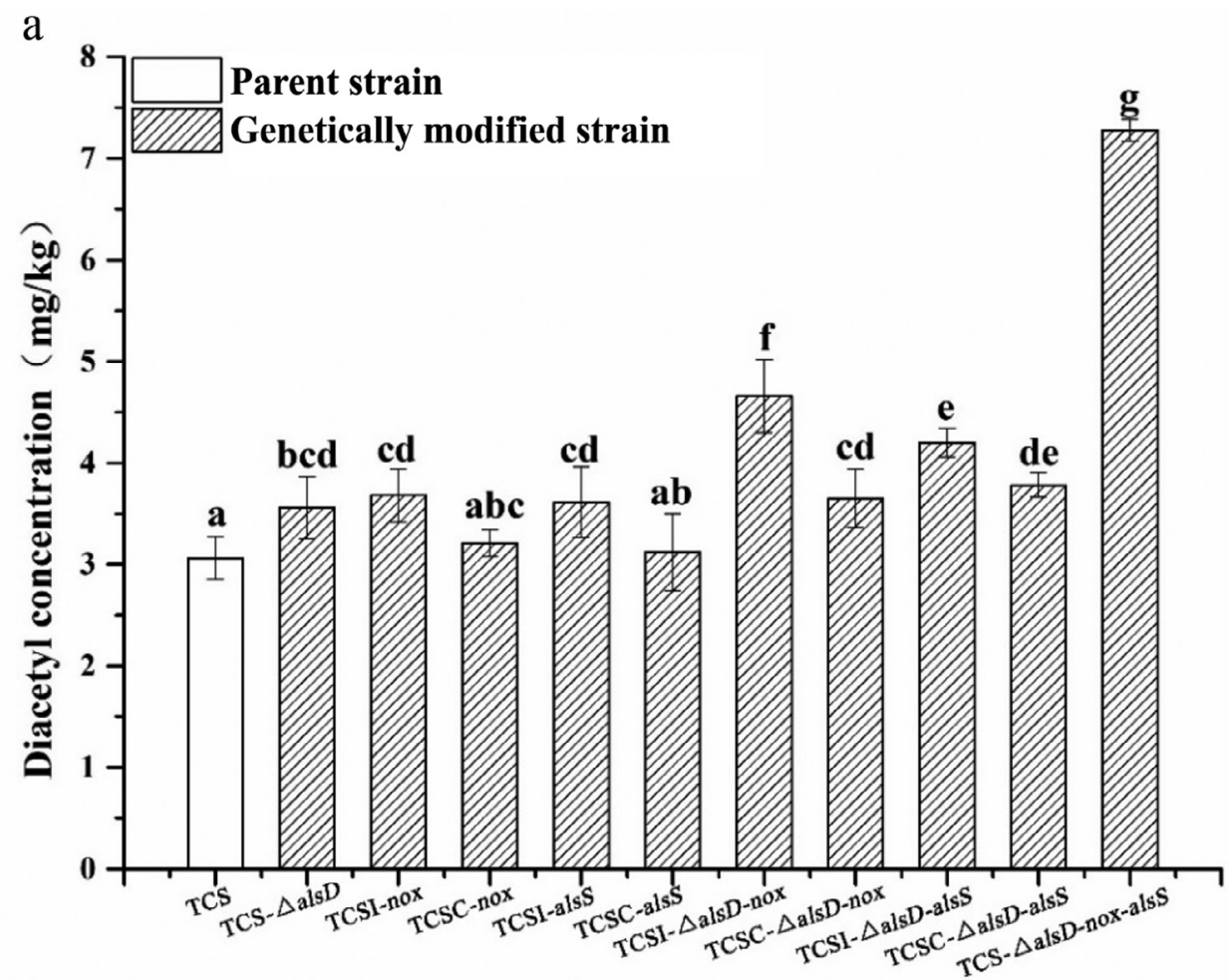

Strains

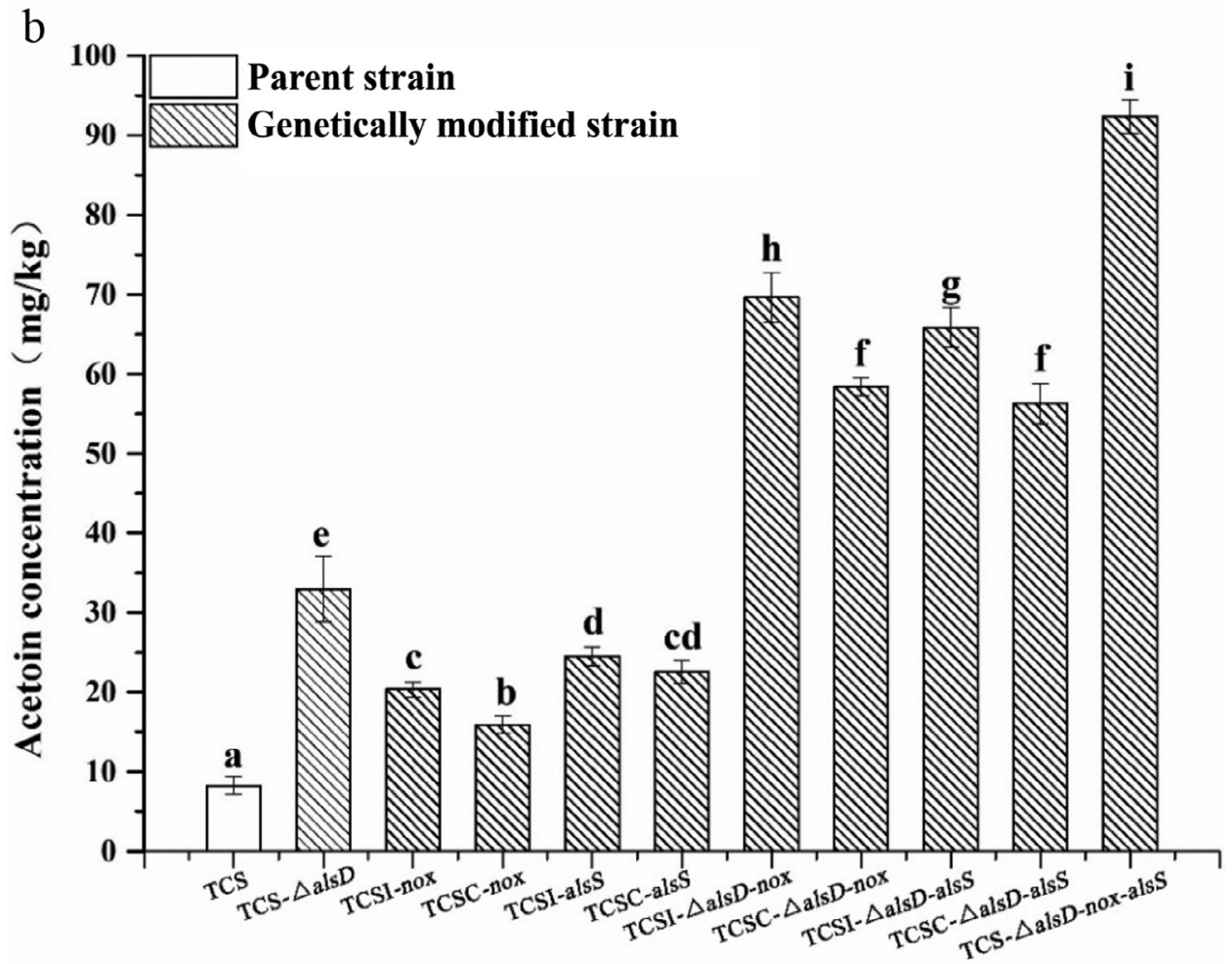

Strains

Figure 4. Diacetyl (a) and acetoin (b) production in milk fermented by wild-type and mutant strains of Lacticaseibacillus casei TCS. Strains are described in Table 1. Error bars represent SD based on 3 replicates. Values marked with different letters (a-i) are significantly different $(P$ $<0.05)$. 
concentrations 2.38 times and 11.19 times higher, respectively, than those yielded by strain TCS.

\section{DISCUSSION}

Three genes, nox, als $S$, and alsD, are known to be important for the biosynthesis of diacetyl and acetoin during the production of fermented milk (Wang et al., 2019a). However, the effects of these genes on diacetyl and acetoin production by $L$. casei have been unclear. In this study, we analyzed the synergistic effects of various modifications of these key genes in L. casei TCS on the yield of diacetyl and acetoin in fermented milk.

Recombinant strains with a single-gene modification produced more diacetyl and acetoin than the original strain during milk fermentation, and this was accompanied by increased expression levels and activities of key enzymes involved in the synthesis of diacetyl and acetoin. Of these recombinant strains, L. casei TCSInox, which had inducible nox gene overexpression, gave the highest yield of diacetyl in fermented milk and had the highest level of NOX activity. Glycolytic flux in $\mathrm{LAB}$ is affected by the redox balance between $\mathrm{NAD}^{+}$ and NADH. It is known that NOX uses NADH to provide an additional pathway for the regeneration of $\mathrm{NAD}^{+}$under aerobic conditions, resulting in the diversion of pyruvate from the NADH-independent pathway and the formation of diacetyl (Lopez de Felipe and Gaudu, 2009; Guo et al., 2012). The overexpression of nox regulates the ratio of $\mathrm{NADH} / \mathrm{NAD}^{+}$, which inhibits other pathways of pyruvate metabolism. Thus, nox gene overexpression directs a greater metabolic flow to pathways of diacetyl and acetoin biosynthesis, greatly increasing their production (Lopez de Felipe and Hugenholtz, 2001). In a similar study, Hugenholtz et al. (2000) changed an $\mathrm{NADH} / \mathrm{NAD}^{+}$ratio by overexpressing the nox gene, which resulted in $80 \%$ of pyruvate being redirected toward the production of diacetyl and acetoin.

Furthermore, the recombinant $L$. casei TCSI-alsS (with inducible als $S$ gene expression) had higher levels of ALS activity and acetoin production than the original strain and the constitutive $L$. casei TCSC-alsS. In similar studies, Dorau et al. (2019) expressed ALS in a metabolically optimized L. lactis strain, which led to the efficient biotransformation of pyruvate into high titers of $\alpha$-acetolactate. Gao et al. (2014) overexpressed the alsS gene in Bacillus subtilis, which led to increased production of diacetyl $(0.57 \mathrm{~g} / \mathrm{L}$, compared with $0.01 \mathrm{~g} / \mathrm{L}$ by non-overexpressed $B$. subtilis). In the current study, the highest acetoin concentration was found in milk fermented by the recombinant $L$. casei TCS- $\Delta$ als $D$, which had its als $D$ gene knocked out. It is possible that knocking out the alsD gene inhibited the reaction of $\alpha$-acetolactate decarboxylase, which may have increased the metabolic flux of diacetyl (Zhang et al., 2015). This would have resulted in more diacetyl being reduced to the downstream product, acetoin (Xiao and Lu, 2014).

Diacetyl and acetoin concentrations were higher in milk fermented by double-gene recombinant strains than in milk fermented by the original strain or singlegene recombinant strains. For example, L. casei TCSI$\Delta$ alsD-nox, which had inducible nox gene expression and the alsD gene knocked out, yielded the highest concentrations of diacetyl and acetoin. This indicates that the adjusted $\mathrm{NADH} / \mathrm{NAD}^{+}$ratio resulting from knocking out als $D$ effectively increased metabolic flux for diacetyl and acetoin production. Hao et al. (2017) knocked out the alsD gene and simultaneously overexpressed the alsS gene in a Bacillus sp., resulting in a diacetyl yield of $1.94 \mathrm{~g} / \mathrm{L}$. Furthermore, similar to the results of single-gene modification, the recombinant strain with inducible expression produced higher concentrations of diacetyl and acetoin than the corresponding recombinant strains with constitutive expression, as evidenced by increased NOX and ALS activities.

When the 3 genes nox, alsS, and alsD were comodified, the yields of diacetyl and acetoin in milk fermented with the resulting recombinant strain were much higher than the yields in milk fermented with strains with single or double genetic modifications. Thus, multiple genetic modifications had a synergistic effect on flavor enhancement. Consistent with previous reports (Hugenholtz et al., 2000; Hao et al., 2017), the overexpression of nox or alsS, as a result of knocking out alsD, led to recombinant strains that exhibited greatly increased diacetyl concentrations. Thus, changes in a single gene may not be sufficient to direct the metabolic flux toward diacetyl and acetoin production, because their precursors, such as $\alpha$-acetolactate and pyruvate, are at the center of the metabolic pathway (Chen et al., 2017).

Notably, our previous study found that the concentrations of diacetyl and acetoin in fermented milk have optimum ranges (Tian et al., 2020), and that excessive concentrations can be perceived by consumers as imparting undesirable flavors. Thus, an optimal flavor is generated by an appropriate concentration of these 2 compounds, which should be accessible by LAB fermentation.

\section{CONCLUSIONS}

In summary, different recombinant strains of $L$. casei TCS were constructed by overexpressing nox and alsS and knocking out alsD. The concentrations of diacetyl and acetoin were higher in milk fermented by the recom- 
binant strains than in milk fermented by the original strain, as determined by various indicators. The modification of multiple genes had a synergistic effect, greatly increasing the biosynthesis of diacetyl and acetoin by $L$. casei during fermented milk production. Our work will support further advances in flavor production during milk fermentation. In particular, although genetically engineered strains are not yet available for food production, this study provides guidance for the subsequent screening and application of relevant strains.

\section{ACKNOWLEDGMENTS}

This research was sponsored by the National Natural Science Foundation of China (no. 31771943, 31972197; Beijing). The authors have not stated any conflicts of interest.

\section{REFERENCES}

Chen, C., L. Wang, H. Yu, and H. Tian. 2020. The local transcriptional regulators SacR1 and SacR2 act as repressors of fructooligosaccharides metabolism in Lactobacillus plantarum. Microb. Cell Fact. 19:161. https://doi.org/10.1186/s12934-020-01403-3.

Chen, C., S. Zhao, G. Hao, H. Yu, H. Tian, and G. Zhao. 2017. Role of lactic acid bacteria on the yogurt flavour: A review. Int. J. Food Prop. 20(Suppl. 1):S316-S330. https://doi.org/10.1080/10942912 .2017.1295988.

Chen, C., F. Zhou, J. Ren, L. Ai, Y. Dong, Z. Wu, Z. Liu, W. Chen, and B. Guo. 2014. Cloning, expression and functional validation of a $\beta$-fructofuranosidase from Lactobacillus plantarum. Process Biochem. 49:758-767. https://doi.org/10.1016/j.procbio.2014.02.013.

Cheng, H. 2010. Volatile flavor compounds in yogurt: A review. Crit. Rev. Food Sci. Nutr. 50:938-950. https://doi.org/10.1080/ 10408390903044081.

Chiocchetti, G. M., C. Jadán-Piedra, V. Monedero, M. Zúñiga, D. Vélez, and V. Devesa. 2019. Use of lactic acid bacteria and yeasts to reduce exposure to chemical food contaminants and toxicity. Crit. Rev. Food Sci. Nutr. 59:1534-1545. https://doi.org/10.1080/ 10408398.2017.1421521.

D'Angelo, L., J. Cicotello, M. Zago, D. Guglielmotti, A. Quiberoni, and V. Suárez. 2017. Leuconostoc strains isolated from dairy products: Response against food stress conditions. Food Microbiol. 66:28-39. https://doi.org/10.1016/j.fm.2017.04.001.

Dorau, R., L. Chen, J. Liu, P. R. Jensen, and C. Solem. 2019. Efficient production of $\alpha$-acetolactate by whole cell catalytic transformation of fermentation-derived pyruvate. Microb. Cell Fact. 18:217. https: //doi.org/10.1186/s12934-019-1271-1.

Fernandez-Garcia, E., and J. U. McGregor. 1994. Determination of organic acids during the fermentation and cold storage of yogurt. J. Dairy Sci. 77:2934-2939. https://doi.org/10.3168/jds.S0022 $-0302(94) 77234-9$.

Gao, X., N. Xu, S. Li, and L. Liu. 2014. Metabolic engineering of Candida glabrata for diacetyl production. PLoS One 9:e89854. https:/ /doi.org/10.1371/journal.pone.0089854.

Güler, Z. 2007. Changes in salted yoghurt during storage. Int. J. Food Sci. Technol. 42:235-245. https://doi.org/10.1111/j.1365-2621 2006.01505.x.

Guo, T., J. Kong, L. Zhang, C. Zhang, and S. Hu. 2012. Fine tuning of the lactate and diacetyl production through promoter engineering in Lactococcus lactis. PLoS One 7:e36296. https://doi.org/10 .1371/journal.pone.0036296.

Hao, W., F. Ji, J. Wang, Y. Wang, Y. Zhang, and Y. Bao. 2017. Metabolic engineering of Bacillus sp. for diacetyl production. Pro- cess Biochem. 58:69-77. https://doi.org/10.1016/j.procbio.2017.04 015 .

Holtzclaw, W. D., and L. F. Chapman. 1975. Degradative acetolactate synthase of Bacillus subtilis: Purification and properties. J. Bacteriol. 121:917-922. https://doi.org/10.1128/jb.121.3.917-922.1975.

Hugenholtz, J., M. Kleerebezem, M. Starrenburg, J. Delcour, W. de Vos, and P. Hols. 2000. Lactococcus lactis as a cell factory for highlevel diacetyl production. Appl. Environ. Microbiol. 66:4112-4114. https://doi.org/10.1128/AEM.66.9.4112-4114.2000.

Kawai, S., S. Mori, T. Mukai, and K. Murata. 2004. Cytosolic NADP phosphatases I and II from Arthrobacter sp. strain KM: Implication in regulation of $\mathrm{NAD}^{+} / \mathrm{NADP}^{+}$balance. J. Basic Microbiol. 44:185-196. https://doi.org/10.1002/jobm.200310362.

Li, F., Y. Shi, J. Zhang, J. Gao, and Y. Zhang. 2018. Cloning, expression, characterization and homology modeling of a novel waterforming NADH oxidase from Streptococcus mutans ATCC 25175. Int. J. Biol. Macromol. 113:1073-1079. https://doi.org/10.1016/j ijbiomac.2018.03.016.

Li, N., Y. Huang, Z. Liu, C. You, and B. Guo. 2015. Regulation of EPS production in Lactobacillus casei LC2W through metabolic engineering. Lett. Appl. Microbiol. 61:555-561. https://doi.org/10 .1111 /lam.12492.

Liu, M., A. Nauta, C. Francke, and R. J. Siezen. 2008. Comparative genomics of enzymes in flavor-forming pathways from amino acids in lactic acid bacteria. Appl. Environ. Microbiol. 74:4590-4600. https://doi.org/10.1128/AEM.00150-08.

Liu, S., Y. Han, and Z. Zhou. 2011. Lactic acid bacteria in traditional fermented Chinese foods. Food Res. Int. 44:643-651. https://doi .org/10.1016/j.foodres.2010.12.034.

Lo, R., V. T. T. Ho, N. Bansal, and M. S. Turner. 2018. The genetic basis underlying variation in production of the flavour compound diacetyl by Lactobacillus rhamnosus strains in milk. Int. J. Food Microbiol. 265:30-39. https://doi.org/10.1016/j.ijfoodmicro.2017 .10 .029 .

Lopez de Felipe, F, and P. Gaudu. 2009. Multiple control of the acetate pathway in Lactococcus lactis under aeration by catabolite repression and metabolites. Appl. Microbiol. Biotechnol. 82:11151122. https://doi.org/10.1007/s00253-009-1897-8.

Lopez de Felipe, F., and J. Hugenholtz. 2001. Purification and characterization of the water forming $\mathrm{NADH}$-oxidase from Lactococcus lactis. Int. Dairy J. 11:37-44. https://doi.org/10.1016/S0958 $-6946(01) 00031-0$

Papagianni, M. 2012. Metabolic engineering of lactic acid bacteria for the production of industrially important compounds. Comput. Struct. Biotechnol. J. 3:e201210003. https://doi.org/10.5936/csbj 201210003.

Raveschot, C., B. Cudennec, B. Deracinois, M. Frémont, M. Vaeremans, J. Dugersuren, S. Demberel, D. Drider, P. Dhulster, F. Coutte, and C. Flahaut. 2020. Proteolytic activity of Lactobacillus strains isolated from Mongolian traditional dairy products: A multiparametric analysis. Food Chem. 304:125415. https://doi.org/10 .1016/j.foodchem.2019.125415.

Shiby, V. K., and H. M. Mishra. 2013. Fermented milks and milk products as functional foods - A review. Crit. Rev. Food Sci. Nutr. 53:482-496. https://doi.org/10.1080/10408398.2010.547398.

Sieuwerts, S., F. A. de Bok, J. Hugenholtz, and J. E. T. van Hylckama Vlieg. 2008. Unraveling microbial interactions in food fermentations: From classical to genomics approaches. Appl. Environ. Microbiol. 74:4997-5007. https://doi.org/10.1128/AEM.00113-08.

Sieuwerts, S., D. Molenaar, S. A. F. T. van Hijum, M. Beerthuyzen, M. J. A. Stevens, P. W. M. Janssen, C. J. Ingham, F. A. M. de Bok, W. M. de Vos, and J. E. T. van Hylckama Vlieg. 2010. Mixed-culture transcriptome analysis reveals the molecular basis of mixed-culture growth in Streptococcus thermophilus and Lactobacillus bulgaricus. Appl. Environ. Microbiol. 76:7775-7784. https: //doi.org/10.1128/AEM.01122-10.

Smit, G., B. A. Smit, and W. J. Engels. 2005. Flavour formation by lactic acid bacteria and biochemical flavour profiling of cheese products. FEMS Microbiol. Rev. 29:591-610. https://doi.org/10 $.1016 /$ j.fmrre.2005.04.002 
Sodini, I., F. Remeuf, S. Haddad, and G. Corrieu. 2004. The relative effect of milk base, starter, and process on yogurt texture: A review. Crit. Rev. Food Sci. Nutr. 44:113-137. https://doi.org/10 $.1080 / 10408690490424793$.

Song, X., H. Huang, Z. Xiong, L. Ai, and S. Yang. 2017. CRISPRCas9 ${ }^{\mathrm{D} 10 \mathrm{~A}}$ nickase-assisted genome editing in Lactobacillus casei. Appl. Environ. Microbiol. 83:e01259-17. https://doi.org/10.1128/ AEM.01259-17.

Sørvig, E., G. Mathiesen, K. Naterstad, V. G. Eijsink, and L. Axelsson. 2005. High-level, inducible gene expression in Lactobacillus sakei and Lactobacillus plantarum using versatile expression vectors. Microbiology (Reading) 151:2439-2449. https://doi.org/10 .1099/mic.0.28084-0.

Tamime, A. Y. 2002. Fermented milks: A historical food with modern applications-A review. Eur. J. Clin. Nutr. 56(Suppl. 4):S2-S15. https://doi.org/10.1038/sj.ejcn.1601657.

Tian, H., Y. Shen, H. Yu, Y. He, and C. Chen. 2017. Effects of 4 probiotic strains in coculture with traditional starters on the flavor profile of yogurt. J. Food Sci. 82:1693-1701. https://doi.org/10 $.1111 / 1750-3841.13779$.

Tian, H., Y. Shi, Y. Zhang, H. Yu, H. Mu, and C. Chen. 2019. Screening of aroma-producing lactic acid bacteria and their application in improving the aromatic profile of yogurt. J. Food Biochem. 43:e12837. https://doi.org/10.1111/jfbc.12837.

Tian, H., B. Yu, H. Yu, and C. Chen. 2020. Evaluation of the synergistic olfactory effects of diacetyl, acetaldehyde, and acetoin in a yogurt matrix using odor threshold, aroma intensity, and electronic nose analyses. J. Dairy Sci. 103:7957-7967. https://doi.org/ 10.3168/jds.2019-17495.

Wang, Y., W. Sun, S. Zheng, Y. Zhang, and Y. Bao. 2019a. Genetic engineering of Bacillus sp. and fermentation process optimizing for diacetyl production. J. Biotechnol. 301:2-10. https://doi.org/ 10.1016/j.jbiotec.2019.05.308

Wang, J., T. Wu, X. Fang, and Z. Yang. 2019b. Manufacture of lowfat Cheddar cheese by exopolysaccharide-producing Lactobacillus plantarum JLK0142 and its functional properties. J. Dairy Sci. 102:3825-3838. https://doi.org/10.3168/jds.2018-15154.

Xiao, Z., and J. R. Lu. 2014. Strategies for enhancing fermentative production of acetoin: A review. Biotechnol. Adv. 32:492-503. https://doi.org/10.1016/j.biotechadv.2014.01.002.

Yang, T., Z. Rao, X. Zhang, M. Xu, Z. Xu, and S. T. Yang. 2017. Metabolic engineering strategies for acetoin and 2,3-butanediol production: Advances and prospects. Crit. Rev. Biotechnol. 37:990-1005. https://doi.org/10.1080/07388551.2017.1299680.

Zhang, L., Y. Zhang, Q. Liu, L. Meng, M. Hu, M. Lv, K. Li, C. Gao, P. Xu, and C. Ma. 2015. Production of diacetyl by metabolically engineered Enterobacter cloacae. Sci. Rep. 5:9033. https://doi.org/ 10.1038/srep09033.

Zhang, X., R. Zhang, T. Bao, T. Yang, M. Xu, H. Li, Z. Xu, and Z. Rao. 2013. Moderate expression of the transcriptional regulator ALsR enhances acetoin production by Bacillus subtilis. J. Ind. Microbiol. Biotechnol. 40:1067-1076. https://doi.org/10.1007/s10295 $-013-1303-5$

\section{ORCIDS}

Huaixiang Tian () https://orcid.org/0000-0002-6097-809X

Haiyan Yu @ https://orcid.org/0000-0001-6472-1366

Bei Wang ๑ https://orcid.org/0000-0001-5407-7810

Chen Chen 은 https://orcid.org/0000-0002-4885-393X 